\title{
INDIRECT EFFECTS IN A PLANKTONIC DISEASE SYSTEM
}

\author{
Z. RAPTI, T. E. STEWART, B. MUELlER-BRENNAN, J. H. KAVOURAS, C. E. CÁCERES
}

\begin{abstract}
Indirect effects, both density- and trait-mediated, have been known to act in tandem with direct effects in the interactions of numerous species. They have been shown to affect populations embedded in competitive and mutualistic networks alike. In this work, we introduce a four-dimensional system of ordinary differential equations and investigate the interplay between direct density-effects and density- and trait-mediated indirect effects that take place in a yeast parasite-zooplankton host-incompetent competitor system embedded in a food web which also includes resources and predators. Among our main findings is the demonstration that indirect effects cause qualitative and quantitative changes almost indistinguishable from direct effects and the theoretical verification of the fact that the effects of direct and indirect mechanisms cannot be disentangled. Our results underpin the conclusions of past studies calling for comprehensive models that incorporate both direct and indirect effects to better describe field data.
\end{abstract}

Z. Rapti and B. Mueller-Brennan, Department of Mathematics, University of Illinois at Urbana-Champaign, Urbana, IL 61801, T. E. Stewart and C. E. Cáceres, School of Integrative Biology, University of Illinois at Urbana-Champaign, Urbana, IL 61801, J. H. Kavouras, Department of Biology, Lewis University, Romeoville, IL 60446

Date: August 22, 2017. 